Supplementary information for

\title{
High-throughput single cell analysis reveals the heterogeneity and dynamics of QDots-uptake and response in macrophages
}

Yuanyuan Wang ${ }^{1,4}$, Ligang $\mathrm{Hu}^{2,4}$, Tian Xia ${ }^{3}$, Xinglei Yao ${ }^{1}$, Yongguang Yin ${ }^{1,4}$, Fengbang Wang ${ }^{1,4}$, maoyong Song ${ }^{1,4 *}$, Guibin Jiang 2,4

${ }^{1}$ Key Laboratory of Environmental Nanotechnology and Health Effects, Research Center for Eco-Environmental Sciences, Chinese Academy of Sciences, Beijing, 100085, China

${ }^{2}$ State Key Laboratory of Environmental Chemistry and Ecotoxicology, Research Center for Eco-Environmental Sciences, Chinese Academy of Sciences, Beijing, 100085, China

${ }^{3}$ Division of Nanomedicine, Department of Medicine, University of California Los Angeles, Los Angeles, CA 90095 , United States

${ }^{4}$ University of Chinese Academy of Sciences, Beijing, 100049, China

* Email: mysong@rcees.ac.cn

The SI contains all supporting figures that are mentioned in the main text. 
a



C

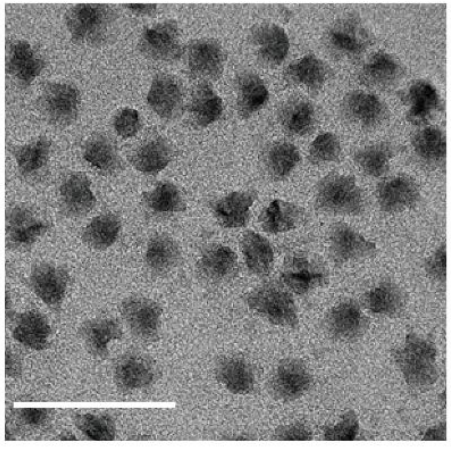

b

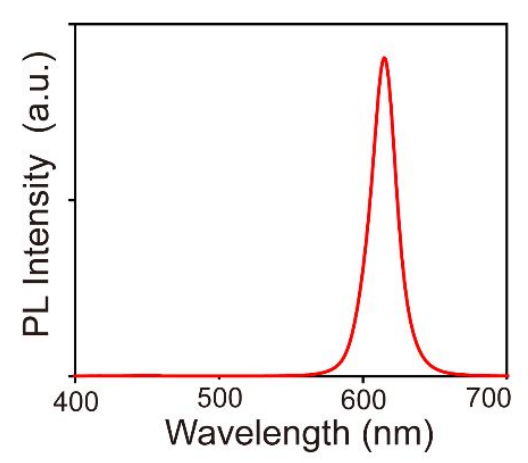

d

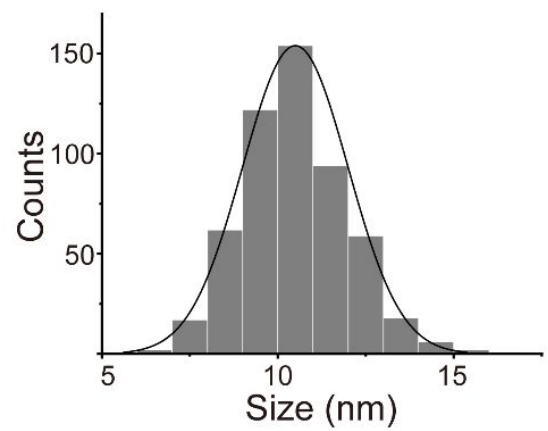

Fig. S1 Characterization of $\mathrm{CdSe} / \mathrm{ZnS}$ QDots. a A schematic depicting the surface properties of QDots. b Fluorescence emission spectrum of QDots with detection wavelength at $450 \mathrm{~nm}$. c TEM images of QDots. Scale bar is $50 \mathrm{~nm}$. d The size distribution of QDots. The histograms were obtained by randomly counting 300 particles in TEM images. 




Fig. S2 Schematic representation of the experimental single cell analysis. About $1 \times 10^{6}$ cells were imaged within a circle well of a 96-well microplate by automated fluorescent imaging system. Red is QDots and blue is nuclear (stained with Hoechst 33342) in the single cell. 

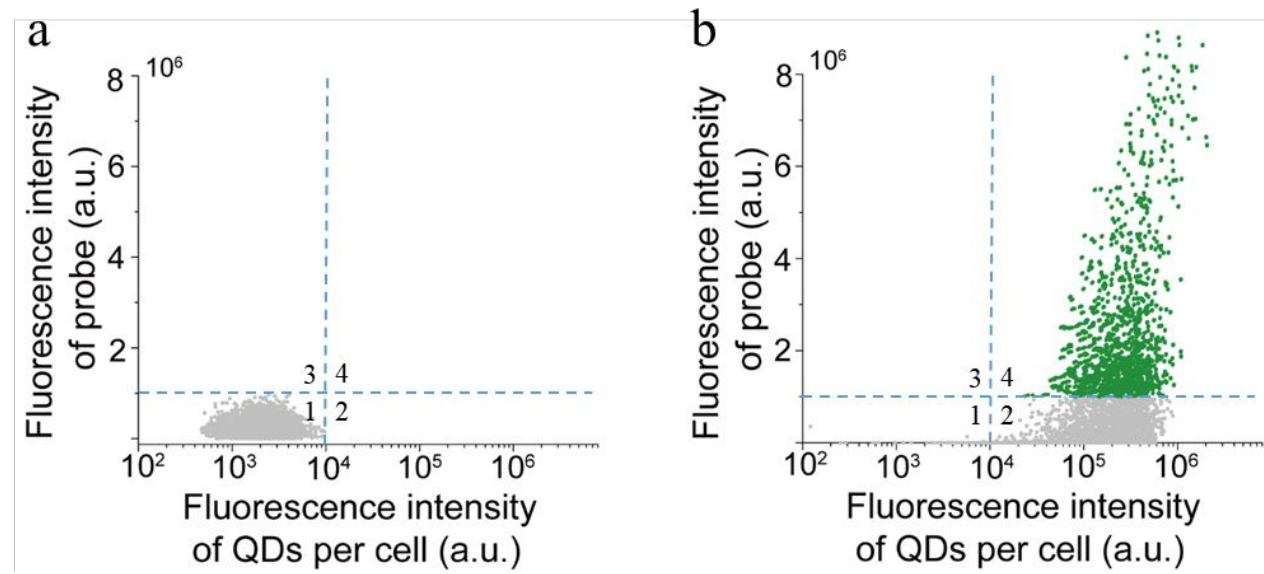

Fig. S3 The "positive-responding" is determined according to the cell response in the control (a) and QDot-treated (b) groups. Cells in each group are divided into four subpopulations based on the values of cell response (vertical axis) and intercellular QDots (horizontal axis). Cells in area 4 are "positive-responding” to QDot exposure. 


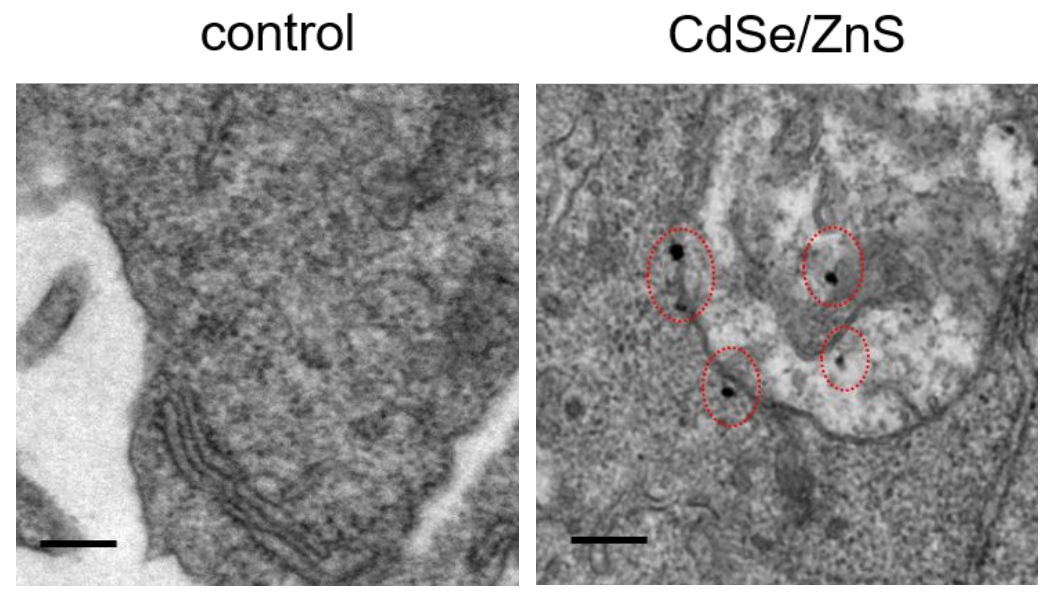

Fig. S4 TEM images showing the uptake of QDots in J774A.1 macrophage. Red circles represent the intracellular QDots. Scale bar is $200 \mathrm{~nm}$. 


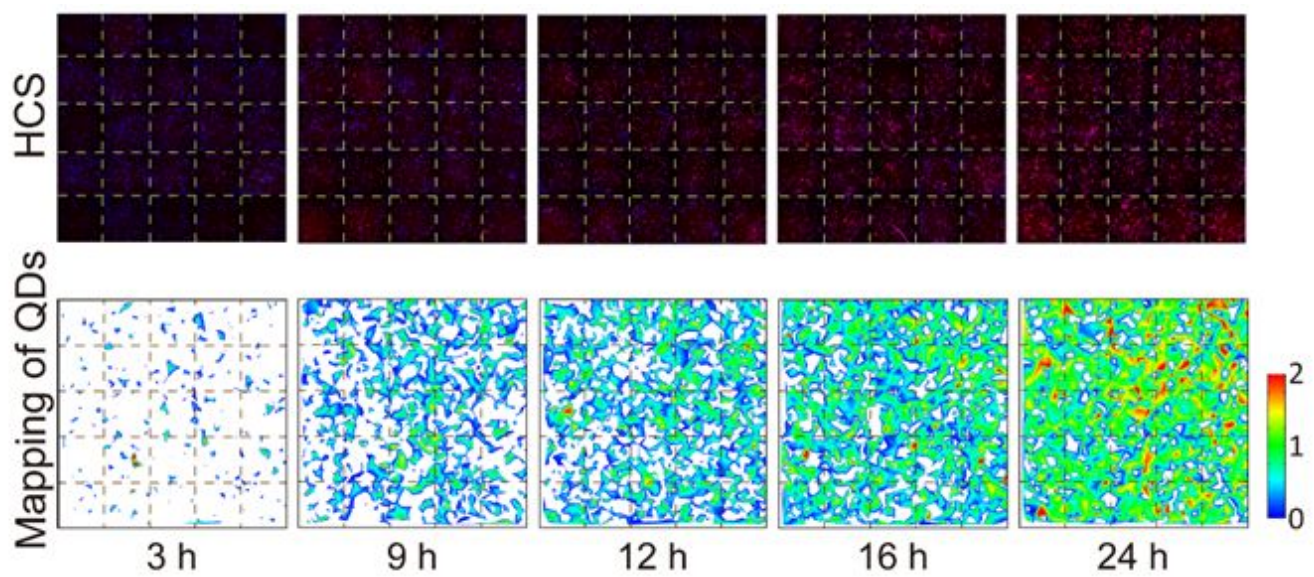

Fig. S5 Spatial and temporal distribution of intracellular QDots. Cells were continuously incubated with $100 \mathrm{nM}$ QDots. Top row is representative HCS images of J774 A.1 macrophage. Bottom row is the hotspot for the distribution of intracellular QDots. The color from blue to red corresponds to logarithm of relative fluorescence intensity of QDots. 

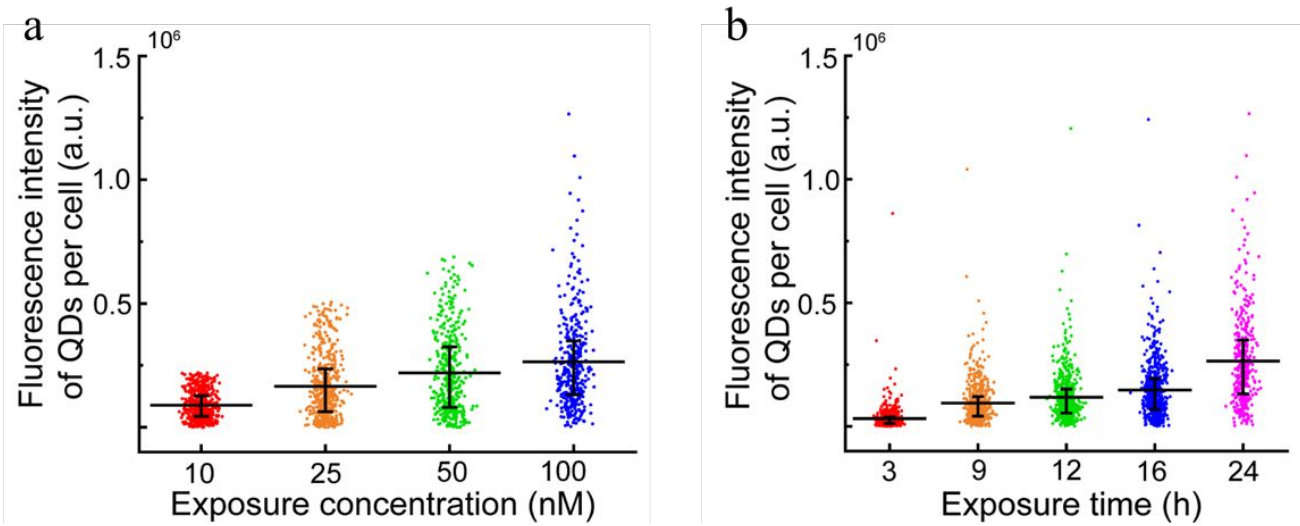

Fig. S6 Scatter interval box plot for the cell distribution based on the fluorescence intensity of intracellular QDots. Each dot represents 100 cells. (a) Cells were exposed to $10,25,50$, and $100 \mathrm{nM}$ QDots for $24 \mathrm{~h}$, respectively. (b) Cells were continuously exposed to $100 \mathrm{nM}$ QDots. 

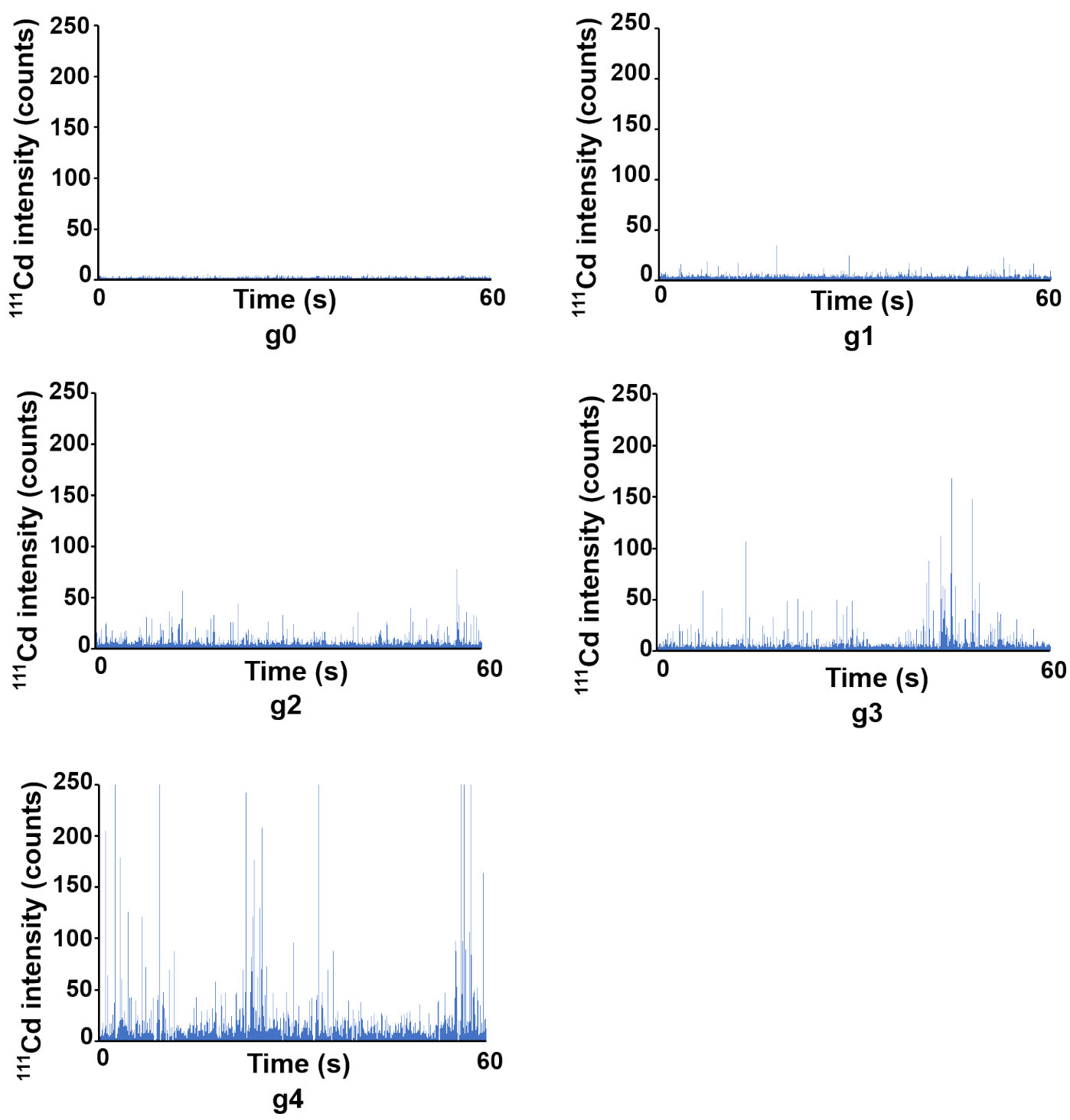

Fig. S7 Representative images of raw mass spectra of cells obtained from group g0 to g4 using SC-ICP-MS. 


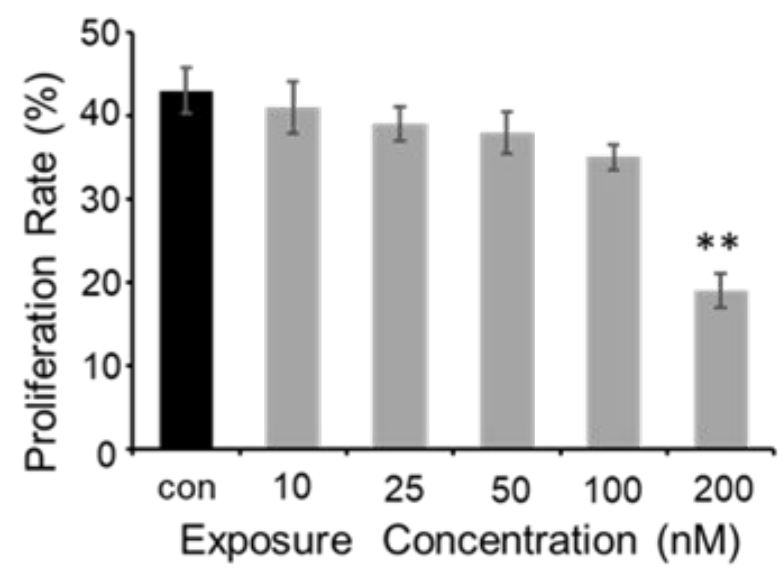

Fig. S8 Viability of macrophages after 24 h exposure to QDots. 